\section{(6) OPEN ACCESS}

\title{
Neutrophil microvesicles resolve gout by inhibiting C5a-mediated priming of the inflammasome
}

\author{
Arun Cumpelik, ${ }^{1,3}$ Barbara Ankli, ${ }^{2}$ Daniel Zecher, ${ }^{1,3,4}$ Jürg A Schifferli, ${ }^{1,3}$
}

\begin{abstract}
Handling editor Tore K Kvien
- Additional material is published online only. To view please visit the journal online (http://dx.doi.org/10.1136/ annrheumdis-2015-207338).

1 Department of Biomedicine, University Hospital Basel, Basel, Switzerland

${ }^{2}$ Department of Rheumatology, University Hospital Basel, Basel, Switzerland

${ }^{3}$ Department of Medicine, University Hospital Basel, Basel, Switzerland ${ }^{4}$ Department of Nephrology, University Hospital Regensburg, Regensburg, Germany
\end{abstract}

\section{Correspondence to}

Professor Jürg A Schifferli, Department of Biomedicine, University Hospital Basel, Hebelstrasse 20, Basel CH-4031, Switzerland; j.schifferli@unibas.ch

DZ and JAS contributed equally.

Received 26 January 2015 Revised 24 June 2015 Accepted 14 July 2015 Published Online First 5 August 2015

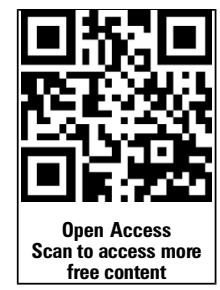

CrossMark

To cite: Cumpelik $A$ Ankli B, Zecher D, et al. Ann Rheum Dis 2016;75:

1236-1245.

\section{ABSTRACT}

Objectives Gout is a highly inflammatory but selflimiting joint disease induced by the precipitation of monosodium urate (MSU) crystals. While it is well established that inflammasome activation by MSU mediates acute inflammation, little is known about the mechanism controlling its spontaneous resolution. The aim of this study was to analyse the role of neutrophilderived microvesicles (PMN-Ecto) in the resolution of acute gout.

Methods PMN-Ecto were studied in a murine model of MSU-induced peritonitis using C57BL/6, MerTK ${ }^{-1-}$ and $\mathrm{C} \mathrm{aR}^{-1-}$ mice. The peritoneal compartment was assessed for the number of infiltrating neutrophils (PMN), neutrophil microvesicles (PMN-Ecto), cytokines (interleukin-1 $\beta$, TGF $\beta$ ) and complement factors (C5a). Human PMN-Ecto were isolated from exudates of patients undergoing an acute gouty attack and functionally tested in vitro.

Results C5a generated after the injection of MSU primed the inflammasome for $\mathrm{IL}-1 \beta$ release. Neutrophils infiltrating the peritoneum in response to $\mathrm{C} 5$ a released phosphatidylserine (PS)-positive PMN-Ecto early on in the course of inflammation. These PMN-Ecto in turn suppressed C5a priming of the inflammasome and consequently inhibited IL-1 $\beta$ release and neutrophil influx. PMN-Ecto-mediated suppression required surface expression of the PS-receptor MerTK and could be reproduced using PS-expressing liposomes. In addition, ectosomes triggered the release of TGF $\beta$ independent of MerTK. TGF $\beta$, however, was not sufficient to control acute MSU-driven inflammation in vivo. Finally, PMNEcto from joint aspirates of patients with gouty arthritis had similar anti-inflammatory properties.

Conclusions PMN-Ecto-mediated control of inflammasome-driven inflammation is a compelling concept of autoregulation initiated early on during PMN activation in gout.

\section{INTRODUCTION}

Gout is a highly inflammatory arthritis induced by the precipitation of monosodium urate (MSU) crystals in articular joints. Even without intervention, acute gouty arthritis (GA) usually resolves spontaneously within a few days leaving minimal residual damage to the joint. What drives this timely resolution of gout, however, is not yet clear.

The early inflammatory phase of gout is characterised by the production of the proinflammatory cytokine interleukin-1 $\beta$ (IL-1 $\beta$ ) and the infiltration of neutrophils into the joint space. IL-1 $\beta$ is the principle driving force of gouty inflammation and is released as a consequence of NLRP3 inflammasome assembly and caspase 1 activation. ${ }^{1}$ The underlying mechanism behind the resolution of gout must therefore interfere with the release of IL-1 $\beta$.

Previous studies indicate that the generation of apoptotic leucocytes and their clearance by macrophages may play a key role in resolving gout. ${ }^{2} 3$ The recognition of phosphatidylserine (PS) on the surface of apoptotic cells and the release of TGF $\beta$ upon their clearance are strong anti-inflammatory cues that can suppress an inflammatory response. ${ }^{4}$ PS-positive surfaces can engage the MerTK receptor initiating the transcription of suppressor of cytokine signalling (SOCS) $3 .^{5-7}$ High levels of SOCS3 expression in synovial tissues as well as elevated levels of TGF $\beta$ in the synovial fluids of patients during the resolution phase of gout have been reported. ${ }^{8}$ Little is however known about how and where the inflammasome is regulated.

We previously established that human neutrophils stimulated by complement $\mathrm{C} 5 \mathrm{a}$ or bacterial peptide fMLP (formyl-methionyl-leucyl-phenylalanine) in vitro release microvesicles (ectosomes) from their surface. ${ }^{9}$ These ectosomes express PS and are capable of inducing the release of TGF $\beta$ by monocyte-derived macrophages. In vitro, neutrophil-derived ectosomes have been shown to suppress the response to TLR ligands in monocytederived macrophages and dendritic cells. ${ }^{10}$

The aim of this study was to determine whether the anti-inflammatory effects of ectosomes extend to the NLRP3 inflammasome, whether ectosomes are found in vivo and whether they take part in the early resolution of gouty inflammation.

\section{MATERIALS AND METHODS}

\section{Generation and analysis of PMN-Ecto and BM-Ecto}

Mouse PMN-Ecto were derived from inflamed peritonea. The peritoneal cavity was lavaged with $5 \mathrm{~mL}$ PBS-1\%FCS, 6-10 h after intraperitoneal injection of $3 \mathrm{mg}$ MSU crystals, and the lavage fluid sequentially centrifuged to separate cells $\left(350 \mathrm{~g} / 10^{\prime} /\right.$ $\left.4^{\circ} \mathrm{C}\right)$, cell debris $\left(3000 \mathrm{~g} / 10^{\prime} / 4^{\circ} \mathrm{C}\right)$ and finally PMN-Ecto $\left(50.000 \mathrm{~g} / 40^{\prime} / 4^{\circ} \mathrm{C}\right)$. The final pellet was diluted in $0.2 \mu \mathrm{m}$ filtered buffers and analysed by flow cytometry. PMN-Ecto were counted using Trucount beads (BD Biosciences, Allschwill, Switzerland) as follows: (\% of total microvesicles $/ \%$ of beads $) \times($ absolute number of beads $) \times(\%$ of annexin $\mathrm{V}^{+}, \mathrm{Gr}-1^{+}$double positive events). Mouse bone marrow-derived ectosomes (BM-Ecto) were prepared from bone marrow cells obtained by flushing femurs and tibias of B6 mice. Erythrocytes 
were removed by hypotonic lysis and the remaining myeloid cells stimulated with $1 \mu \mathrm{M}$ fMLP (Sigma, St Louis, Missouri, USA) or $10 \mathrm{ng} / \mathrm{mL}$ mouse recombinant C5a (BD Pharmingen) in RPMI for $30 \mathrm{~min}$ at $37^{\circ} \mathrm{C}$. The supernatant was sequentially centrifuged as above. Where indicated, BM-Ecto were stained with $5 \mu \mathrm{M}$ CFSE (Molecular Probes, Zug, Switzerland). Human PMN-Ecto were isolated by sequentially centrifuging joint exudates from patients with gout or control patients with osteoarthritis (OA). Flow cytometry was performed using a CyanADP cytometer (Beckman Coulter, Nyon, Switzerland). Data were analysed using FlowJo Software (TreeStar, San Jose, California, USA).

\section{Cell culture conditions, ELISA and western blot}

Resident peritoneal macrophages were harvested by peritoneal lavage and plated at $2 \times 10^{6} /$ well. To achieve full inflammasome activation in vitro, cells were first primed with $10 \mathrm{ng} / \mathrm{mL}$ ultrapure LPS (Invivogen, Toulouse, France) or $10 \mathrm{ng} / \mathrm{mL}$ mouse recombinant $\mathrm{C} 5 \mathrm{a}(\mathrm{BD})$ for $10 \mathrm{~h}$, washed and subsequently stimulated with $100 \mu \mathrm{g} / \mathrm{mL}$ MSU. Macrophages were treated with BM-Ecto $\left(1 \times 10^{8} \mathrm{BM}\right.$-Ecto $/ 2 \times 10^{6}$ macrophages $)$ or liposomes containing either phosphatidylserine (PS) or control phosphatidylcholine (PC) $\left(1 \times 10^{8}\right.$ liposomes $/ 2 \times 10^{6}$ macrophages $)$ for 10 min either prior to LPS priming or prior to MSU stimulation. Cell extracts and cell culture supernatants were prepared for western blotting as described previously. ${ }^{11}$ SOCS3 protein expression was analysed by densitometry and normalised to actin using Image Lab software (Biorad, Munich, Germany).

Cell culture supernatants and peritoneal lavages were analysed for IL-1 $\beta$, IL-10, IL-1Ra and TGF $\beta$ using the IL-1 $\beta$, IL-10 OptEIA (BD Pharmingen), IL-1Ra (R\&D, DY480) and TGF $\beta 1$ (eBiosciences, Vienna, Austria) ELISA kits, respectively, according to the manufacturer's instructions.

\section{Experimental peritonitis}

Mice were treated with an intraperitoneal injection of $2 \times 10^{7} \mathrm{BM}-$ Ecto/PMN-Ecto or comparable amounts of PS or control PC liposomes $2 \mathrm{~h}$ prior to intraperitoneal stimulation with $3 \mathrm{mg} \mathrm{MSU}$ crystals. Control groups received Ecto followed by $0.9 \% \mathrm{NaCl}$ or received the stimulus preceded by an injection of $0.9 \% \mathrm{NaCl}$ instead of Ecto. At the indicated time points following induction of peritonitis, mice were sacrificed by $\mathrm{CO}_{2}$-inhalation and peritoneal lavage was performed with $5 \mathrm{~mL}$ PBS-1\% FCS. The lavage fluid was filtered $(70 \mu \mathrm{m})$, washed and contaminating red blood cells removed by hypotonic lysis. Leucocytes were counted with an automated cell counter (Beckman Coulter, Nyon, Switzerland). Peritoneal leucocytes were phenotyped by flow cytometry following surface staining with the indicated antibodies (see online supplementary figure S1A).

For detailed methods see online supplementary material.

\section{RESULTS \\ Bone marrow-derived ectosomes suppress inflammasome activation in vitro}

We first set out to find a source of murine ectosomes using B6 wild type (WT) bone marrow cells as a close approximation of neutrophils. BM-Ecto were isolated by sequentially centrifuging supernatants of bone marrow cells stimulated with fMLP. Flow cytometric analysis identified intact vesicles that were able to retain CFSE. Further characterisation revealed the expression of the neutrophil marker Gr-1 and surface exposure of PS (assessed by annexin $\mathrm{V}$ staining) on approximately $70-80 \%$ of intact BM-Ecto (figure 1A). Consistent with data from human neutrophil-derived ectosomes, ${ }^{9}$ electron microscopy of BM-Ecto preparations revealed round shaped vesicles with a size of 50 $500 \mathrm{~nm}$ (figure 1B).

We next asked whether BM-Ecto suppress MSU-induced inflammasome activation of resident peritoneal macrophages in vitro. Inflammasome activation in vitro is a two-step process that requires priming with a TLR ligand (LPS) $)^{12}$ prior to stimulation with a specific inflammasome activator (MSU) (figure 1C). Whereas upregulation of pro-IL-1 $\beta$ and NALP3 components in cells is the measure of successful priming, release of mature IL- $1 \beta$ along with the caspase 1 subunits $\mathrm{p} 20$ or $\mathrm{p} 10$ defines efficient inflammasome stimulation. ${ }^{1}{ }^{13}{ }^{14}$ To determine whether BM-Ecto interfere with inflammasome activation, BM-Ecto were given to macrophages either prior to LPS priming or prior to stimulation with MSU (figure 1D) once all LPS priming events (pro-IL $\beta$ and NALP3 upregulation) had taken place.

The release of IL-1 $\beta$ into culture supernatants was significantly suppressed when macrophages received BM-Ecto either prior to LPS priming or MSU stimulation (figure 1E), indicating that BM-Ecto acted on both phases of inflammasome activation. Immunoblots of cell extracts (figure 1F) and cell culture supernatants (figure $1 \mathrm{~F}$ ) revealed that incubation of macrophages with BM-Ecto prior to LPS impaired efficient priming (less NALP3 and pro-IL-1 $\beta$ expression) and subsequently rendered macrophages unresponsive to MSU stimulation (no caspase 1 p20/10 and IL-1 $\beta$ release). The addition of BM-Ecto after LPS priming resulted in partial suppression of the MSU response (no caspase 1 p20 and less IL-1ß), consistent with data obtained by ELISA (figure 1E). These results indicated that BM-Ecto contain inflammasome activation in vitro by suppressing LPS priming and MSU stimulation independently of each other.

The anti-inflammatory effects of Ecto have been attributed to their surface expression of $\mathrm{PS}^{15}$ analogous to what has been reported for apoptotic cells. ${ }^{457}$ We therefore asked whether the in vitro effects of BM-Ecto could be reproduced by size-matched liposomes expressing PS. Following our in vitro stimulation protocol (figure 1D) macrophages were treated with equal amounts (approximately $1 \times 10^{8}$ ) of PS or control PC liposomes. While PS liposomes interfered with LPS priming (figure 1G, H, cell extracts) and MSU stimulation (figure 1G, $\mathrm{H}$, cell supernatants), PC liposomes failed to attenuate the inflammasome response at any level. These results suggested that PS is involved in the inhibition of inflammasome activation by BM-Ecto.

\section{C5a and MSU-induced inflammation}

Inflammasome activation in macrophages is functionally limited by low expression of pro-IL-1 $\beta$ and therefore requires some form of priming. ${ }^{12}$ During gout, however, activation of the NLRP3 inflammasome occurs in a sterile environment and has been shown to be independent of Toll-like-receptor $4 .{ }^{16}$ Therefore the use of LPS as a priming agent in vitro does not accurately reflect what occurs in vivo. MSU crystals are known to activate complement by assembling a functional C5 convertase complex at the crystal surface, which results in the generation of active $\mathrm{C} 5 \mathrm{a} .{ }^{17} \mathrm{C} 5 \mathrm{a}$ fragments have been reported to activate NFKB. ${ }^{18-20}$ Given that the injection of MSU alone can trigger IL-1 $\beta$ release, ${ }^{121}$ we hypothesised that C5a generated by MSU is responsible for inflammasome priming in vivo.

MSU crystals generated C5a in the presence of plasma, which in turn primed the inflammasome via the C5aR leading to the release of IL-1 $\beta$ upon MSU stimulation of macrophages in vitro (figure 2A). BM-Ecto were capable of suppressing IL-1 $\beta$ release from macrophages stimulated by MSU in the presence of plasma (figure $2 \mathrm{~B}$ ) and macrophages primed with $\mathrm{C} 5 \mathrm{a}$ and subsequently 
A
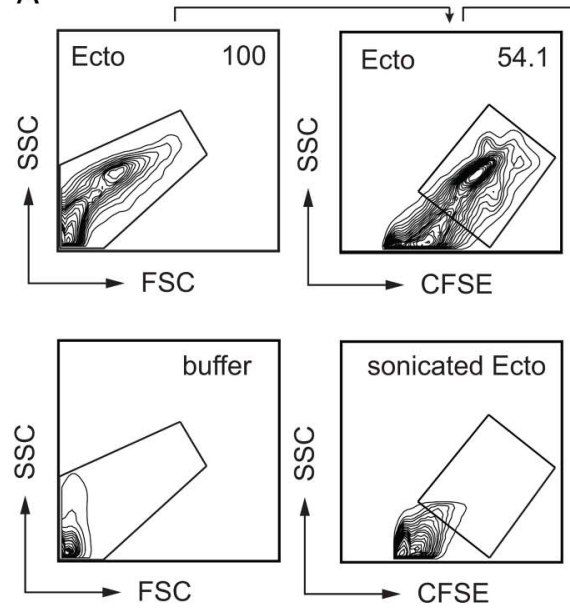

CFSE

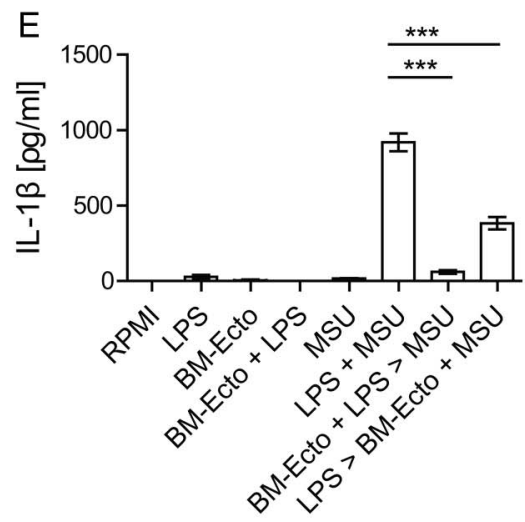

F

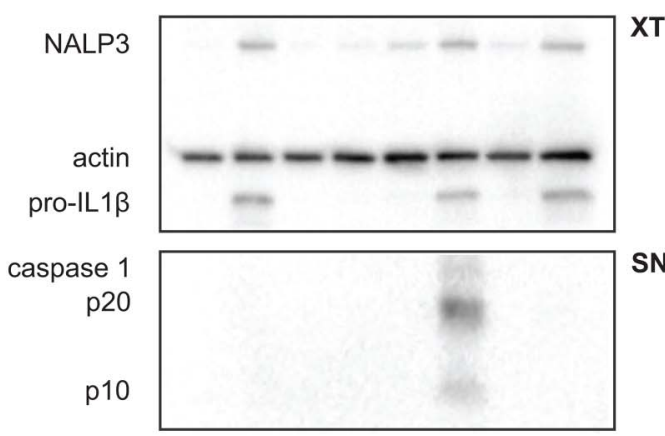

IL1 $\beta$
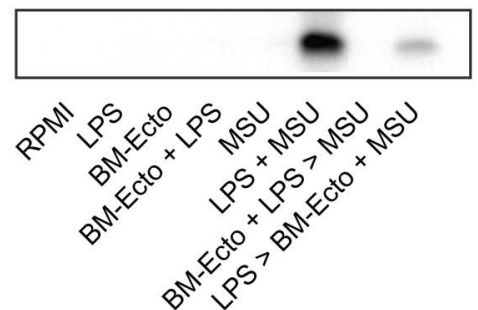

$\mathrm{H}$

caspase

IL1 $\beta$

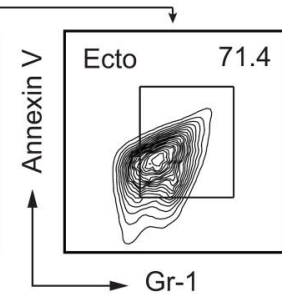

B

C

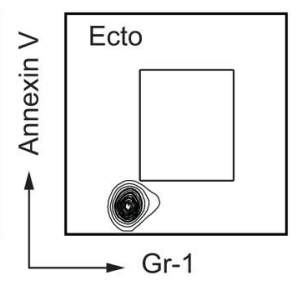

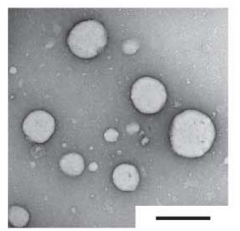

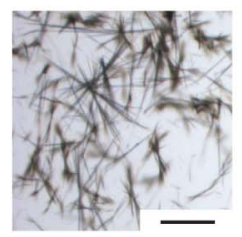

D

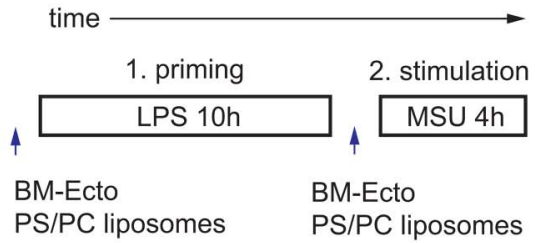

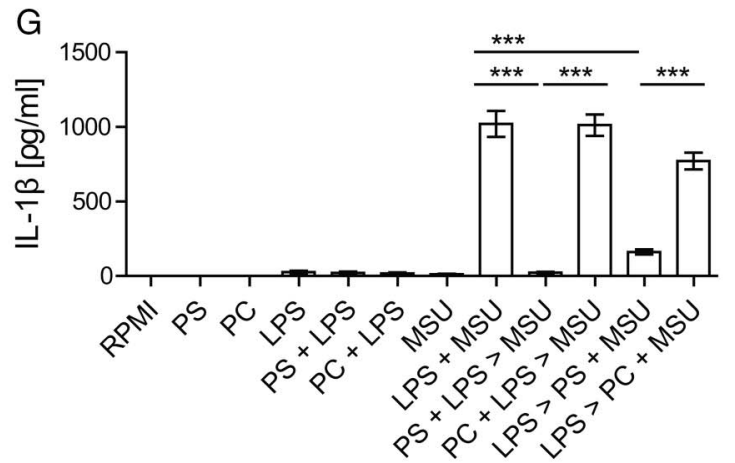

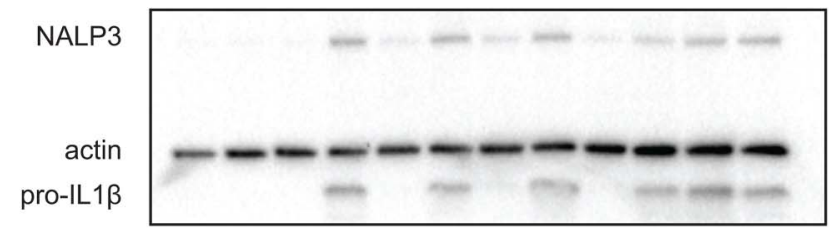

XT

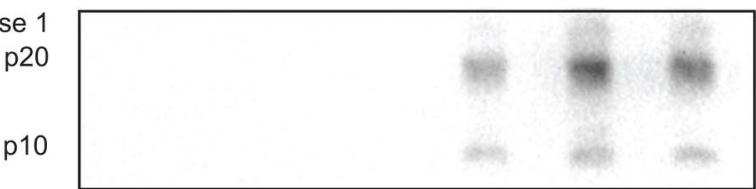

SN

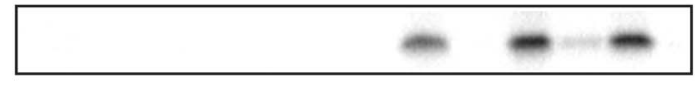

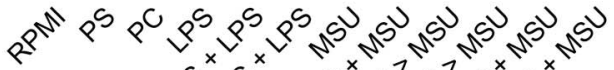

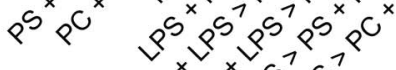

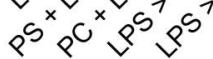

Figure 1 Characterisation and in vitro properties of BM-Ectosomes. (A) Flow cytometric characterisation of bone marrow-derived ectosomes (BM-Ecto). CFSE-positive events (representing intact vesicles) were analysed for surface expression of Gr-1 and phosphatidylserine (PS; using annexin V) (upper lane, left to right). Controls are (lower lane, left to right): annexin buffer alone, CFSE threshold set on sonicated BM-Ecto previously stained with CFSE, BM-Ecto stained with annexin V and IgG1 isotype in PBS. FSC denotes forward scatter, SSC side scatter, respectively. Numbers indicate \% positive BM-Ecto (B) Morphology of BM-Ecto (size bar $100 \mathrm{~nm}$ ) and (C) monosodium urate (MSU) crystals (size bar $50 \mu \mathrm{m}$ ) as determined by transmission electron microscopy and light microscopy, respectively. (D) In vitro stimulation protocol. B6 peritoneal macrophages were primed with LPS for $10 \mathrm{~h}$ and subsequently stimulated with $100 \mu \mathrm{g} / \mathrm{mL}$ MSU for $4 \mathrm{~h}$. BM-Ecto $\left(1 \times 10^{8}\right.$ BM-Ecto $/ 2 \times 10^{6}$ macrophages) were given either prior (BM-Ecto+LPS>MSU) or after (LPS>BM-Ecto+MSU) LPS priming as outlined. Alternatively, PS-liposomes or control PC-liposomes were given instead of BM-Ecto. (E and G) IL-1 $\beta$ in cell culture supernatants determined by ELISA. $n=4$ per group. ( $F$ and $H$ ) Cell extracts (XT) and supernatants (SN) were analysed for the presence of NALP3, pro-IL-1 $\beta$, IL-1 $\beta$ and active caspase 1 (p20 and p10) by western blot. Data in A, F and $\mathrm{H}$ are representative of three independent experiments. ${ }^{* *} \mathrm{p}<0.001$. Mean $\pm \mathrm{SEM}$ is shown. 

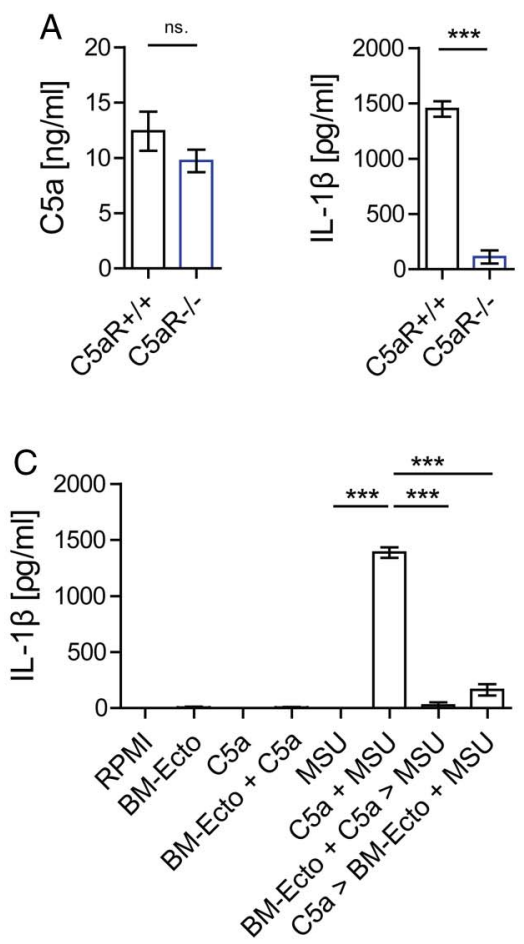

$\mathrm{D}$
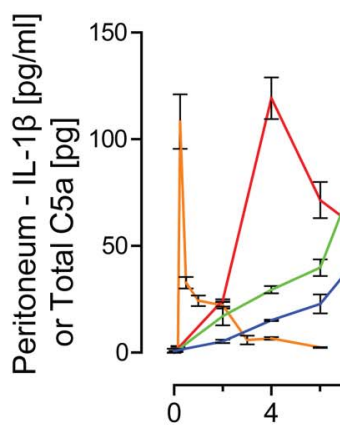

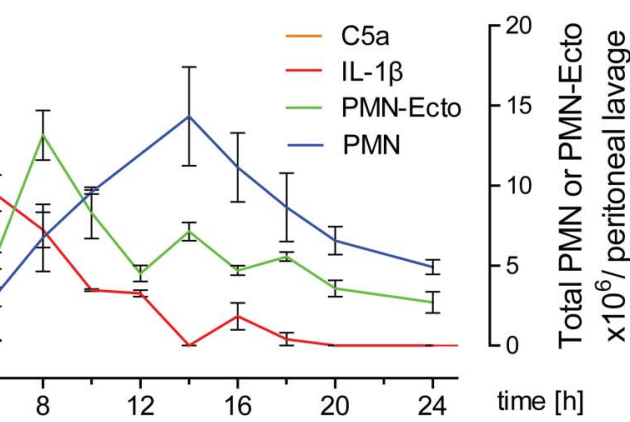

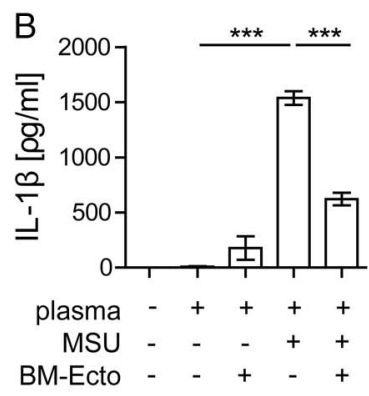
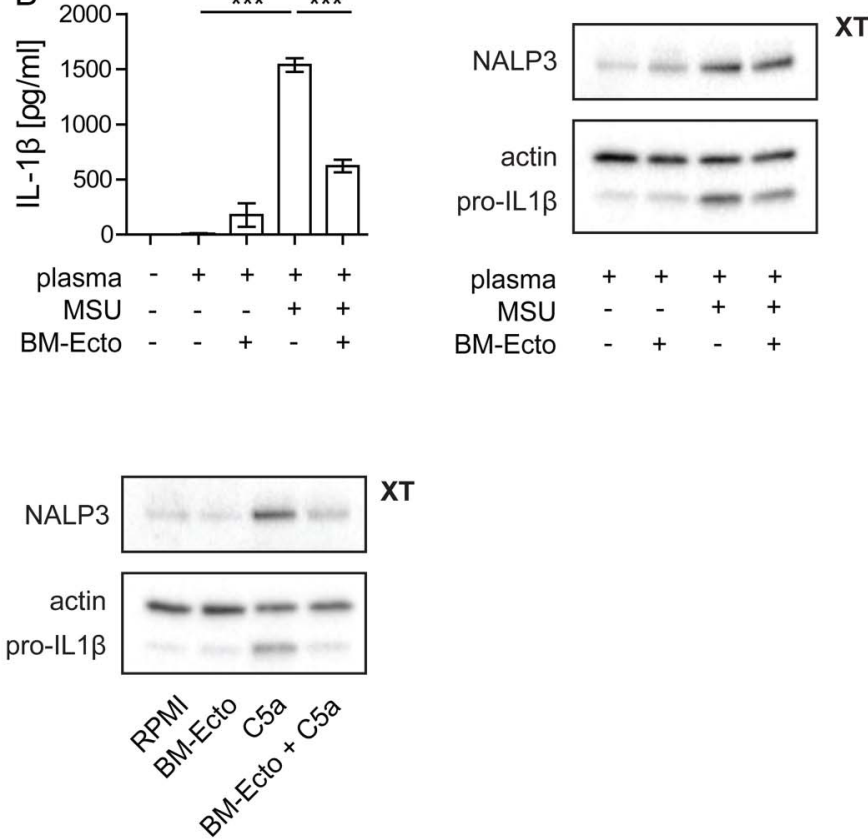

\section{T}

$\mathrm{E}$

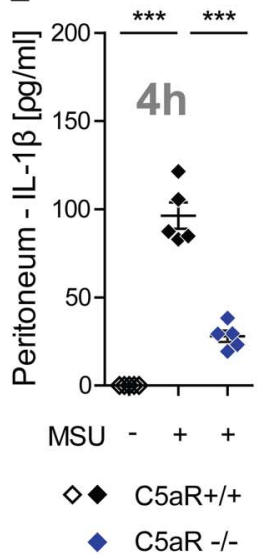

$\mathrm{F}$

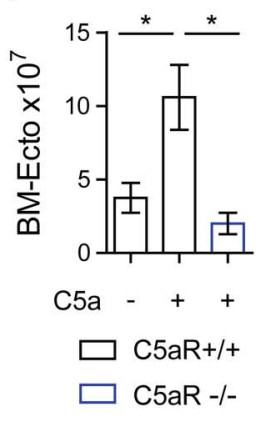

Figure 2 Role of C5a in monosodium urate (MSU)-induced inflammation. (A) Inflammasome activation by MSU is dependent on C5a in vitro. Generation of $\mathrm{C} 5 \mathrm{a}$ and IL-1 $\beta$ release from $\mathrm{C}_{2} \mathrm{R}^{+/+}$or $\mathrm{C}_{5} \mathrm{R}^{-/-}$peritoneal macrophages stimulated with $100 \mu \mathrm{g} / \mathrm{mL}$ MSU in the presence of $25 \%$ plasma for $14 \mathrm{~h}$ in vitro. $\mathrm{n}=6$ per group pooled from two independent experiments. (B and C) BM-Ecto inhibit C5a-mediated inflammasome activation in vitro. (B) BM-Ecto were given to B6 macrophages prior to stimulation with $100 \mu \mathrm{g} / \mathrm{mL}$ MSU in the presence of $25 \%$ plasma for $14 \mathrm{~h}$ or to (C) macrophages primed with $10 \mathrm{ng} / \mathrm{mL}$ recombinant mouse C5a for $10 \mathrm{~h}$ and subsequently stimulated with $100 \mu \mathrm{g} / \mathrm{mL} \mathrm{MSU}$ for $4 \mathrm{~h}$. IL-1 $\beta$ release, pro-IL-1 $\beta$ and NALP3 expression were analysed as in figure 1. $n=6$ per group pooled from two independent experiments (ELISA), western blots representative of two independent experiments. (D) Kinetics of MSU-induced peritonitis. B6 mice received an intraperitoneal injection of $3 \mathrm{mg}$ MSU. At the indicated time points thereafter, peritonea were lavaged and infiltrating cells phenotyped by flow cytometry PMN were identified as $\mathrm{CD}^{+} 5^{+}, \mathrm{CD} 11 \mathrm{~b}^{+}, \mathrm{Ly}_{6 \mathrm{C}}{ }^{+}, \mathrm{Ly}_{6 \mathrm{G}}{ }^{+}$cells. Peritoneal concentrations of C5a and IL-1 $\beta$ were determined by ELISA. $n=4 /$ time point pooled from two independent experiments. (E) Inflammasome activation is C5a-dependent in vivo. Concentration of IL-1 $\beta$ in the peritoneal lavage fluid of $\mathrm{C5}^{\mathrm{R}}{ }^{+/+}$ and $\mathrm{CaR}^{-l-}$ mice $4 \mathrm{~h}$ after injection of $3 \mathrm{mg} \mathrm{MSU} . \mathrm{n}=5$ pooled from two independent experiments. (F) Release of BM-Ecto requires the C5aR. $1 \times 10^{7}$ bone marrow cells were stimulated with $10 \mathrm{ng} / \mathrm{mL}$ recombinant mouse C5a for $30 \mathrm{~min}$ at $37^{\circ} \mathrm{C}$. BM-Ecto were isolated from the supernatant as indicated in Methods. $n=5$ pooled from two independent experiments. ${ }^{*} p<0.05,{ }^{* * *} p<0.001$. Mean $\pm S E M$ is shown.

stimulated with MSU (figure 2C). These results suggested that C5a is essential for inflammasome activation by MSU and that BM-Ecto inhibit C5a-mediated inflammasome priming.

To study inflammasome activation in vivo, we next adopted a murine model of MSU-induced peritonitis. ${ }^{21}$ We first analysed the course of the inflammatory response following intraperitoneal injection of MSU. There was an almost immediate and steep rise of C5a 15 min after introducing MSU into the peritoneum (figure 2D). The generation of C5a was followed by the release of IL-1 $\beta$ in the peritoneum peaking $4 \mathrm{~h}$ after MSU injection (figure 2D). The release of C5a and IL-1 $\beta$ triggered a rise in blood neutrophils (not shown), which then infiltrated the peritoneal compartment reaching a maximum $14 \mathrm{~h}$ after stimulation (figure 2D). In accordance with our in vitro results, the release of IL-1 $\beta$ in response to MSU was significantly impaired in $\mathrm{C} 5 \mathrm{aR}$ deficient mice (figure 2E).

We next determined whether Ecto are released by infiltrating neutrophils during MSU-induced peritonitis. At various time 
points following MSU stimulation, Ecto were isolated from peritoneal lavages by sequential centrifugation. Using flow cytometry, surface staining with annexin $\mathrm{V}$ and anti-Gr-1 identified them as PS-positive microvesicles of neutrophil origin (see online supplementary figure S1B). These neutrophil ectosomes (PMN-Ecto) were found to be present in significant numbers, reaching up to $1.5 \times 10^{7}$ in the peritoneum $8 \mathrm{~h}$ after MSU stimulation (figure 2D). The kinetics of PMN-Ecto suggested that their release is an early event of neutrophil activation. Given that bone marrow cells release BM-Ecto in response to C5a, complement activation is the likely trigger for ectosome release in gout (figure $2 \mathrm{~F}$ ).

\section{Administration of Ecto attenuates MSU-induced inflammation in vivo}

To determine whether Ecto have anti-inflammatory properties in vivo, the peritoneal compartment was pretreated with $2 \times 10^{7}$ Ecto $2 \mathrm{~h}$ prior to the intraperitoneal injection of MSU. The Ecto used to precondition the peritoneum were either BM-Ecto isolated from ex vivo stimulated bone marrow cells or PMN-Ecto isolated from the lavage fluid of MSU-inflamed peritonea. The quantity $\left(2 \times 10^{7}\right)$ of preinjected BM-Ecto and PMN-Ecto corresponded to the maximum number of PMN-Ecto recovered during MSU-induced peritoneal inflammation (figure 2D).

Pretreatment with PMN-Ecto or BM-Ecto resulted in a twofold suppression of IL-1 $\beta$ release in response to MSU (figure $3 \mathrm{~A})$ and subsequently a threefold decrease in the number of infiltrating neutrophils into the peritoneal compartment $14 \mathrm{~h}$ after stimulation (figure $3 \mathrm{~B}$ ). Taken together, these results indicated that Ecto from two independent sources can suppress inflammation induced by MSU.

\section{The in vivo effects of Ecto can be mimicked by liposomes expressing PS}

To confirm that PS liposomes can act as a surrogate for Ecto in vivo, approximately $2 \times 10^{7}$ PS or control PC liposomes were injected intraperitoneally instead of Ecto $2 \mathrm{~h}$ prior to stimulation with MSU. Pretreatment with PS liposomes resulted in suppression of IL-1 $\beta$ (figure $3 \mathrm{C}$ ) and neutrophil influx into the peritoneum (figure 3D) in response to MSU. Injection of control PC liposomes failed to achieve an anti-inflammatory effect.

\section{Suppression by BM-Ecto is MerTK-dependent in vivo}

To directly test the involvement of PS in Ecto-mediated immunosuppression, we next applied our model to mice lacking the MerTK receptor $\left(\mathrm{MerTK}^{-/-}\right)$which is known to bind PS and relay its signal by inducing SOCS3. ${ }^{7}$

The peritonea of $\mathrm{MerTK}^{-/}$and control B6129S (WT) mice were pretreated with BM-Ecto prior to MSU stimulation. Whereas pretreatment of WT mice with BM-Ecto led to suppression of IL-1 $\beta$ release (figure $4 \mathrm{~A}$ ) and neutrophil influx (figure 4B) in the peritoneum, the anti-inflammatory effects of BM-Ecto were absent in $\mathrm{MerTK}^{-/-}$mice. Furthermore, $4 \mathrm{~h}$ after intraperitoneal BM-Ecto injection, we observed a MerTK-dependent induction of SOCS3 in peritoneal macrophages (figure 4C). Of note, inflammation induced by MSU in $\mathrm{MerTK}^{-/-}$mice was significantly higher compared with background-matched WT mice (figure 4A, B), suggesting that
Figure 3 Administration of ectosomes attenuates monosodium urate (MSU)-driven peritoneal inflammation. B6 mice were injected intraperitoneally with $3 \mathrm{mg} \mathrm{MSU}$. Where indicated, mice were preinjected with $2 \times 10^{7}$ BM-Ecto or PMN-Ecto intraperitoneally $2 \mathrm{~h}$ prior to MSU stimulation. Alternatively, mice were preinjected with $75 \mathrm{nM}$ (approximately $2 \times 10^{7}$ ) of phosphatidylserine (PS)-liposomes or phosphatidylcholine (PC)-liposomes. Control groups received BM-/PMN-Ecto or PS-I PC-liposome injections intraperitoneally followed by $\mathrm{NaCl}$ instead of MSU. (A and C) IL-1 $\beta$ in peritoneal lavage fluid was determined by ELISA $4 \mathrm{~h}$ after MSU stimulation. ( $B$ and D) The number of infiltrating PMN into the peritoneum $14 \mathrm{~h}$ after MSU stimulation was determined as indicated in figure 2D. $n=6-8$ per group pooled from at least three independent experiments, ${ }^{*} p<0.05,{ }^{* *} p<0.01$, $* * * p<0.001$. Mean \pm SEM is shown.
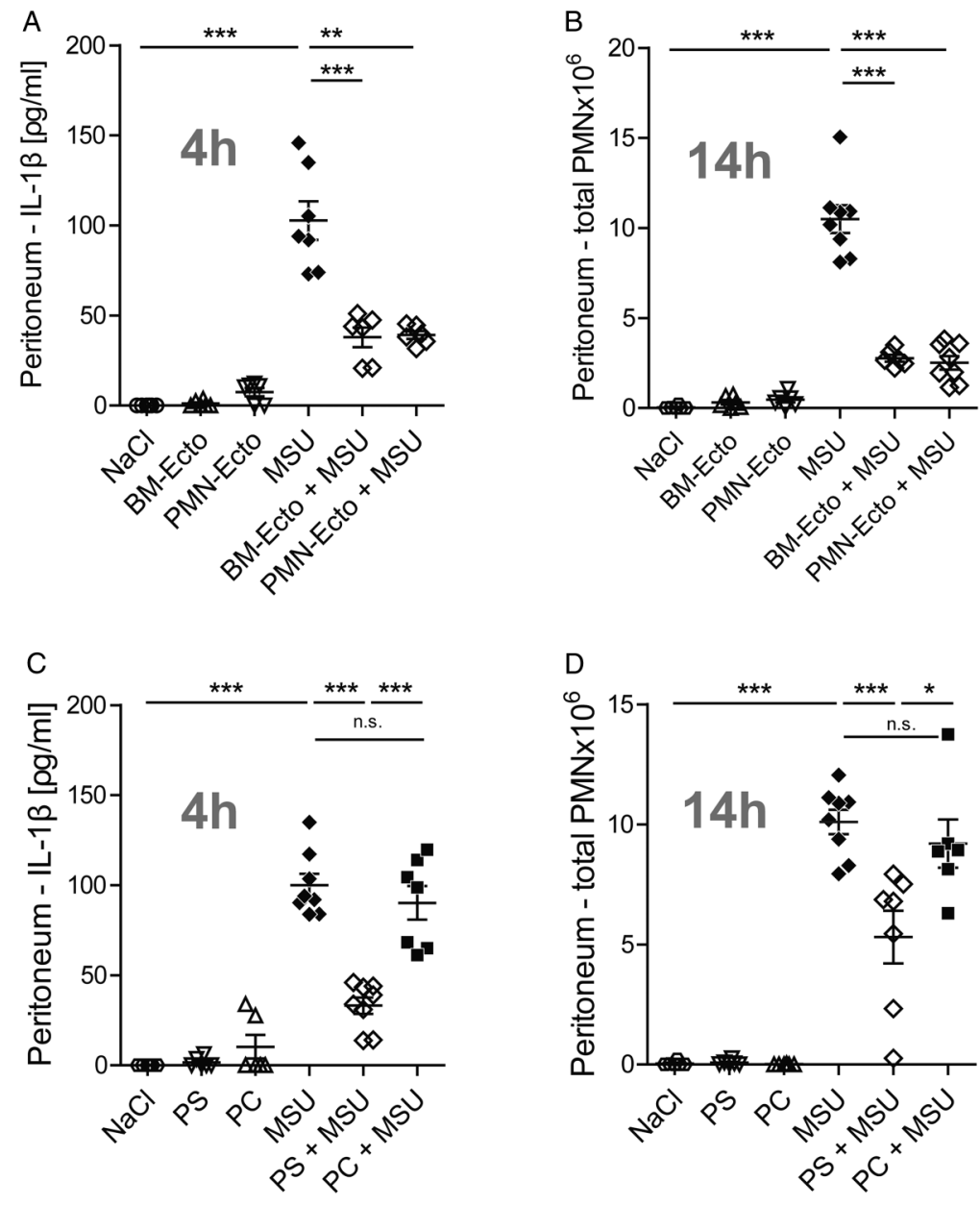
endogenously released PMN-Ecto limit the magnitude of MSU-induced inflammation via MerTK in WT mice.

\section{BM-Ecto induce the release of TGF $\beta$}

It has been suggested that TGF $\beta$ participates in the resolution of gout in its late stages. ${ }^{22}$ Therefore, we next asked whether Ecto induce the release of TGF $\beta$ in vivo. Injection of BM-Ecto induced the release of TGF $\beta$ in the peritoneum (figure $5 \mathrm{~A}$ ). The release of TGF $\beta$ was further enhanced when BM-Ecto primed mice received MSU (figure 5A). Since BM-Ecto themselves were not the source of TGF $\beta$ (figure $5 \mathrm{~B}$ ) and the amount of TGF $\beta$ released in vitro by peritoneal macrophages remained the same regardless of inflammasome activation (figure $5 \mathrm{C}$ ), we hypothesised that the additive effect of MSU and BM-Ecto on TGF $\beta$ release could be due to infiltrating cells responding to BM-Ecto. Indeed, monocytes and neutrophils isolated from MSU inflamed peritonea were able to release TGF $\beta$ in response to BM-Ecto in vitro (figure $5 \mathrm{D}$ ). To determine which of these cells contributes the most to the TGF $\beta$ pool in vivo, resident macrophages, infiltrating monocytes and neutrophils from BM-Ecto-treated peritonea were stained for latency associated peptide (LAP). LAP is part of latent TGF $\beta$ and remains tethered to the surface of macrophages ${ }^{23}$ and monocytes $^{24}$ once TGF $\beta$ is released and cleaved into its active form. Whereas expression of LAP in naive resident peritoneal macrophages $\left(\mathrm{F}_{480}{ }^{+}, \mathrm{CD}_{115^{+}}, \mathrm{Ly}_{6} \mathrm{C}^{-}\right)$was low, LAP progressively increased over time after intraperitoneal injection of BM-Ecto, indicating continuous TGF $\beta$ release (figure 5E). Monocytes and neutrophils are not present in untreated peritonea. Upon intraperitoneal MSU stimulation, however, infiltrating monocytes $\left(\mathrm{Ly}_{6} \mathrm{C}^{+}\right.$, $\mathrm{F}_{480}{ }^{-}, \mathrm{Ly}_{6 \mathrm{G}^{-}}$) and, to a lesser extent, neutrophils $\left(\mathrm{Ly} 6 \mathrm{C}^{+}\right.$, $\mathrm{F}_{480}{ }^{-}, \mathrm{Ly}_{6 \mathrm{G}}{ }^{+}$) upregulated LAP when the peritoneum was pretreated with BM-Ecto prior to MSU (figure 5E).

\section{Ecto suppress MSU-induced peritonitis independent of TGF $\beta$}

We next asked whether TGF $\beta$ was necessary for the suppressive effects of Ecto. In vitro, TGF $\beta$ release was independent of
MerTK (figure 5F). In vivo, significant increases in TGF $\beta$ were consistently measured in the peritonea of WT and $\mathrm{MerTK}^{-/}$ mice pretreated with BM-Ecto (figure 5G). Moreover, PS liposomes did not induce TGF $\beta$ release in vivo (figure $5 \mathrm{H}$ ). Since PS liposomes attenuated the response to MSU (figure 3C, D) without inducing TGF $\beta$ and BM-Ecto failed to inhibit inflammation in $\mathrm{MerTK}^{-1-}$ mice (figure 4A, B) despite releasing TGF $\beta$ (figure 5G), TGF $\beta$ did not seem to be necessary for BM-Ecto-mediated resolution of acute gouty inflammation. To confirm that the effect of BM-Ecto was independent of TGF $\beta$, neutralising anti-TGF $\beta 1$ antibody was given intraperitoneally $30 \mathrm{~min}$ prior to BM-Ecto. In the presence of TGF $\beta 1$-blocking antibody, BM-Ecto still retained their capacity to suppress inflammation (figure 5I). Although the blocking of TGF $\beta 1$ slightly increased neutrophil influx in response to MSU suggesting that TGF $\beta$ may play a role (figure 5I), the injection of recombinant mouse TGF $\beta 1$ instead of BM-Ecto had no effect (figure 5J).

Taken together, these data suggested that Ecto inhibit the acute inflammatory response to MSU predominantly via the PS-MerTK pathway rather than TGF $\beta$. Although Ecto induced the release of TGF $\beta$ by macrophages and monocytes, MerTK alone was necessary and sufficient for Ecto to suppress inflammasome activation in vivo.

\section{PMN-Ecto are present in synovial exudates during gouty inflammation in humans}

Lastly, we sequentially centrifuged synovial exudates of patients with GA and control patients with OA to verify the presence of microvesicles during gouty attacks in humans. Arthrocentesis was performed within 1 day after the onset of symptoms. Using flow cytometry, we found annexin V-positive vesicles expressing the granulocyte marker CD66b and the neutrophil-specific enzyme myeloperoxidase (figure 6A). Electron micrographs (figure 6B) of exudates confirmed the presence of intact vesicles that were approximately $50 \mathrm{~nm}$ in size.
Figure 4 Ecto-mediated immunosuppression requires MerTK in vivo. B6/129S (wild type, WT) or MerTK $^{-1-}$ mice were injected intraperitoneally with $3 \mathrm{mg}$ monosodium urate (MSU). Where indicated, mice were preinjected with $2 \times 10^{7}$ BM-Ecto intraperitoneally $2 \mathrm{~h}$ prior to MSU stimulation. (A) IL-1 $\beta$ was determined $4 \mathrm{~h}$ after MSU stimulation in the peritoneal lavage fluid. (B) The number of infiltrating PMN into the peritoneum $14 \mathrm{~h}$ after MSU stimulation was determined as in figure $2 D$. $n=6-8$ per group, pooled from at least three independent experiments. (C) Suppressor of cytokine signalling (SOCS3) expression in peritoneal macrophages of $\mathrm{B} 6 / 129 \mathrm{~S}$ (WT) and $\mathrm{MerTK}^{-/-}$mice determined by immunoblot $4 \mathrm{~h}$ after intraperitoneal BM-Ecto injection. Expression of SOCS3 in arbitrary units of band intensity normalised to actin. $n=2$ per group pooled from two independent experiments. * $p<0.05$, ${ }^{* *} p<0.05$. Mean \pm SEM is shown. n.s., not significant.
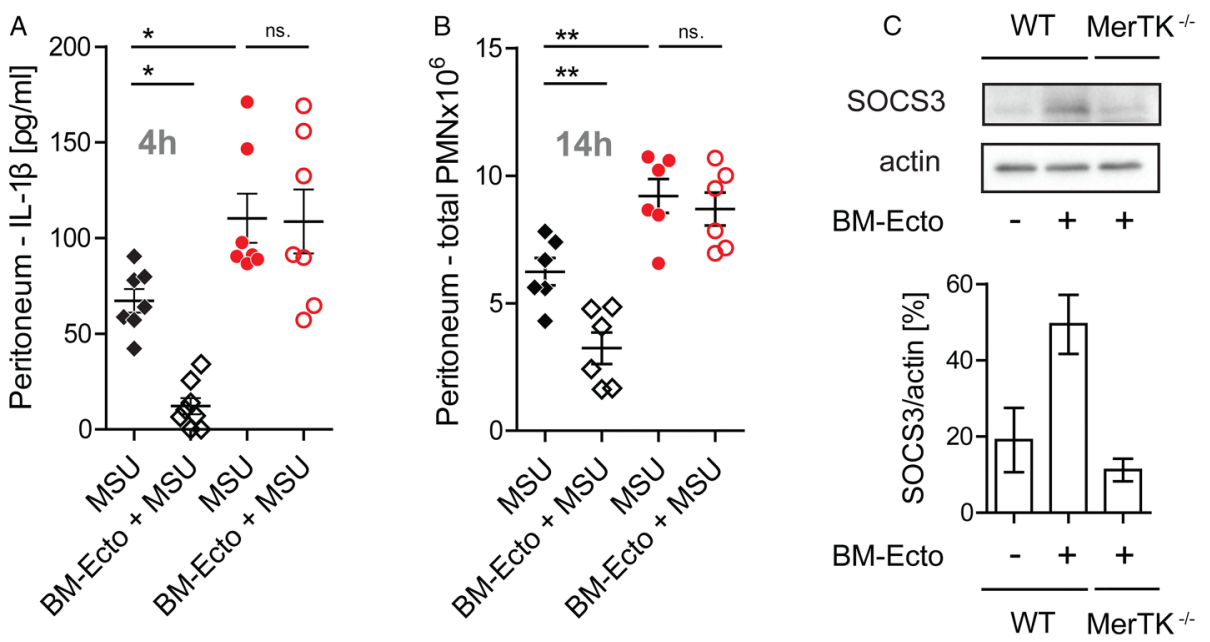

$\diamond$ WT

- 0 MerTK ${ }^{-1}$ 

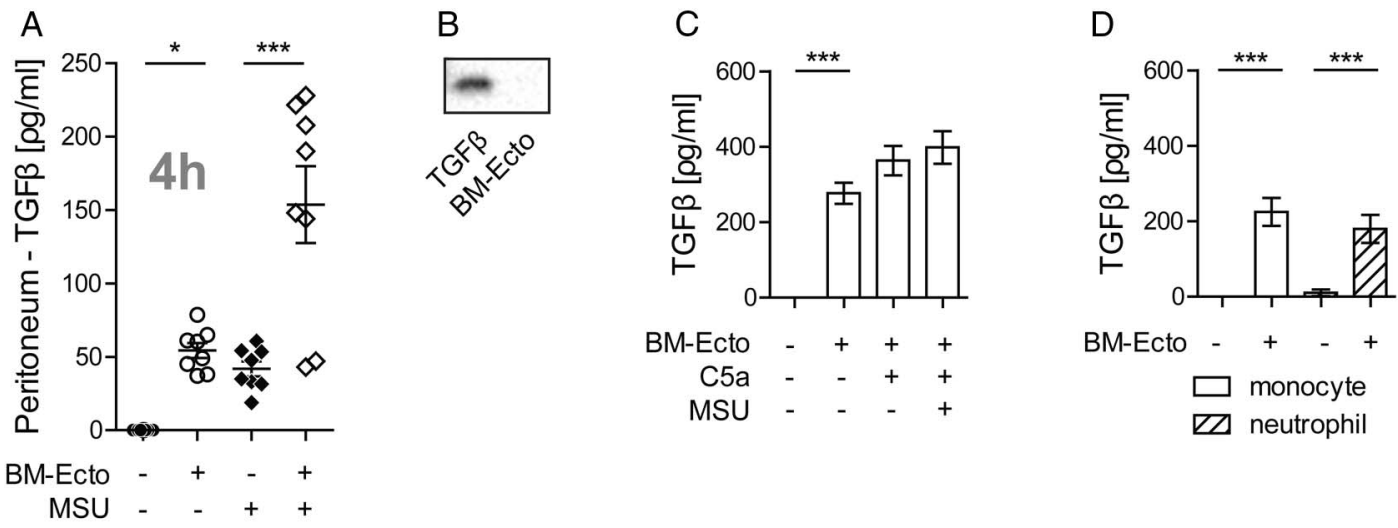

E macrophage

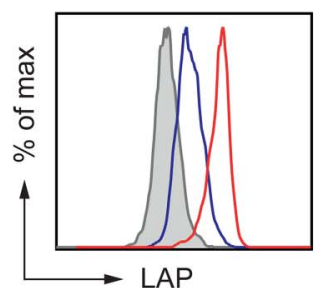

isotype control

untreated

4h - BM-Ecto
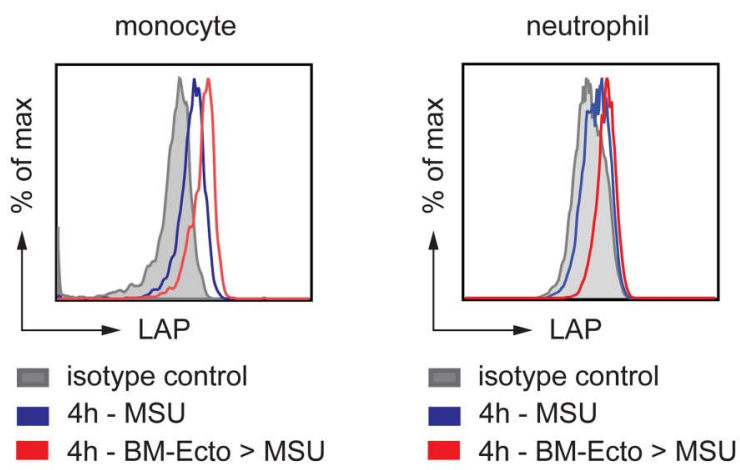

F

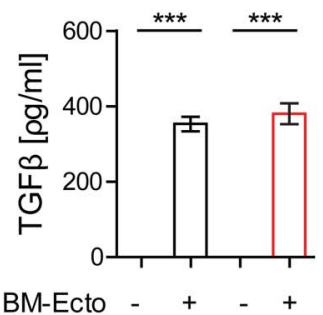

$\square$ WT

$\square \operatorname{MerTK}^{-1-}$

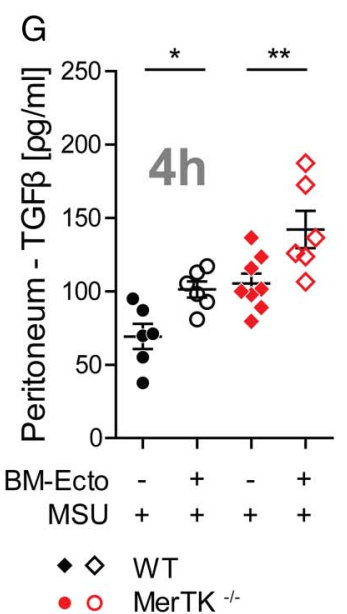

$\mathrm{H}$

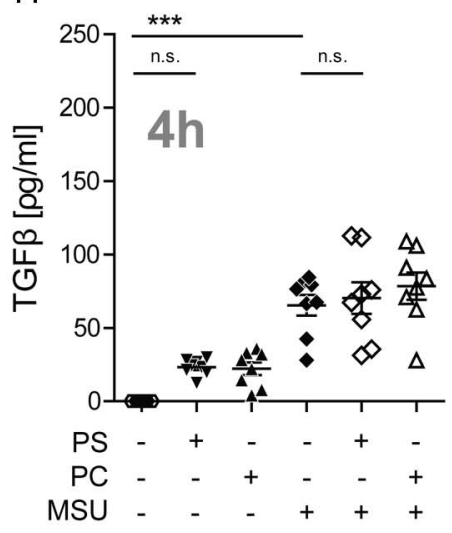

1

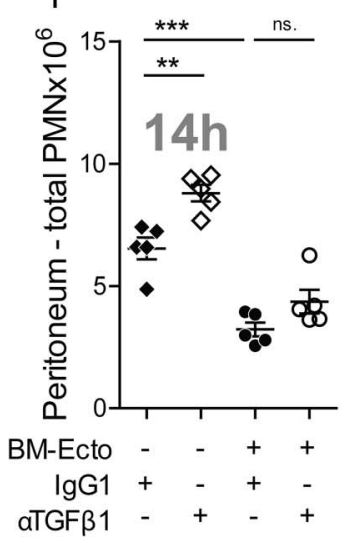

J

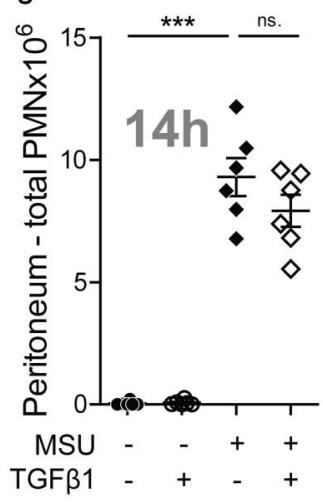

Figure 5 Ecto induce the release of TGF- $\beta$ independent of MerTK. (A) TGF $\beta$ concentration in peritoneal lavage fluids of B6 mice treated as outlined in figure 3 was determined by ELISA. $n=6-8$ pooled from three independent experiments. (B) Ecto are not the source of TGF $\beta$ in vivo. BM-Ecto lysates were assessed for TGF $\beta$ content by immunoblot. Recombinant mouse TGF $\beta$ was used as control. (C-E) Cellular source of TGF $\beta$. Release of TGF $\beta$ by B6 (C) resident peritoneal macrophages or (D) monocytes and neutrophils isolated from monosodium urate (MSU)-inflamed peritonea following treatment with C5a, MSU and/or BM-Ecto in vitro as outlined in figure 2C. (E) Expression of latency associated peptide (LAP) on B6 macrophages, monocytes and neutrophils isolated either $4 \mathrm{~h}$ after intraperitoneal injection of BM-Ecto or $4 \mathrm{~h}$ after intraperitoneal injection of MSU with BM-Ecto pretreatment. Controls received $\mathrm{NaCl}$ intraperitoneally (untreated). ( $\mathrm{F}-\mathrm{H}) \mathrm{TGF} \beta$ release is independent of MerTK. ( $\mathrm{F}$ ) The release of TGF $\beta$ by wild type (WT) and MerTK ${ }^{-1-}$ macrophages treated with BM-Ecto in vitro. $n=6 /$ group. TGF $\beta$ in peritoneal lavage fluids of (G) B6/129S (WT) and MerTK ${ }^{-1-}$ mice treated with BM-Ecto or (H) B6 mice treated with liposomes as outlined in figures 3 and 4 , respectively. $n=6-8 / g r o u p$. (I and J) Effect of TGF $\beta$ in vivo. (I) The effect of neutralising anti-TGF $\beta 1$ antibodies on peritoneal PMN influx using $100 \mu \mathrm{g}$ anti-TGF $\beta 1$ injected intraperitoneally $15 \mathrm{~min}$ prior to MSU or 15 min prior to BM-Ecto pretreatment. (J) The effect of $1 \mu \mathrm{g}$ recombinant mouse TGF $\beta 1$ injected intraperitoneally instead of BM-Ecto prior to MSU stimulation. ${ }^{*} p<0.05,{ }^{*} p<0.01,{ }^{* * *} p<0.001$. Mean $\pm S E M$ is shown. n.S., not significant.

The amount of PMN-Ecto isolated from gout exudates correlated with the number of infiltrating PMN (figure 6C) and GA exudates had significantly higher numbers of PMN-Ecto compared to OA exudates (figure 6D). Recovery of neutrophilderived ectosomes from synovial exudates of patients during gouty inflammation suggested that PMN-Ecto release occurs and is relevant in vivo.

In vitro, PMN-Ecto from gout exudates (Gout-Ecto) were able to induce the release of TGF $\beta$ from human monocyte-derived macrophages (figure $6 \mathrm{E}$ ) and inhibited the release of IL- $1 \beta$ by 
macrophages (figure 6F) treated as outlined in figure 1D. Furthermore, in the presence of human plasma, MSU crystals were able to simultaneously prime and stimulate the inflammasome. Consistently, MSU failed to prime the inflammasome in the presence of heat inactivated or C5-blocked plasma, confirming that priming is C5-dependent (figure 6G). Finally, Gout-Ecto suppressed IL-1 $\beta$ release in macrophages stimulated by MSU in the presence of human plasma (figure $6 \mathrm{H}$ ).

\section{DISCUSSION}

The major findings of the present study are related to $\mathrm{C} 5 \mathrm{a}$ and PMN-Ecto release in gout. C5a generated by MSU is responsible for priming the inflammasome and consequently for the release of IL-1 $\beta$. Furthermore, C5a induces PMN-Ecto release by infiltrating neutrophils and these ectosomes in turn limit inflammasome priming. Ectosomes achieve their anti-inflammatory effect by engaging the MerTK receptor. The regulation induced by PMN-Ecto starts almost immediately after the influx of cells into the peritoneum, indicating that the control of inflammation starts much earlier than presumed until now. Interestingly, the very cells that are responsible for acute inflammation (ie, neutrophils), act also as its regulator due to the shedding of ectosomes.

The release of PMN-Ecto is an early phenomenon of neutrophil activation. ${ }^{9}$ In our gout model, PMN-Ecto were released as early as $2 \mathrm{~h}$ after intraperitoneal injection of MSU and their concentration peaked at $8 \mathrm{~h}$. To analyse their effect on gouty inflammation, ectosomes were given intraperitoneally prior to MSU injection. The number of ectosomes injected was
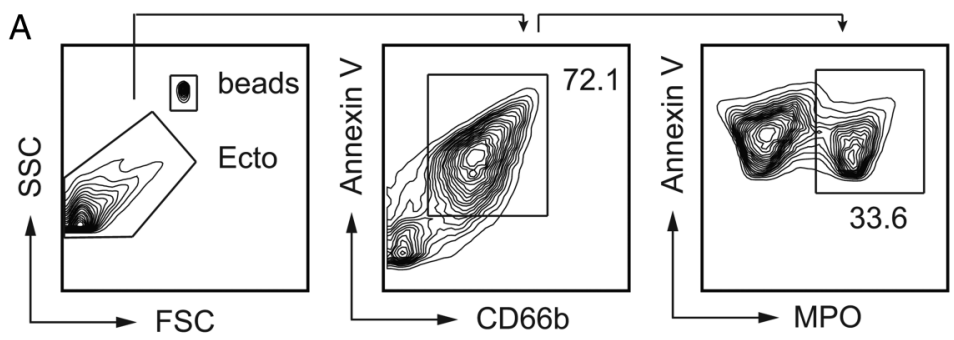

B
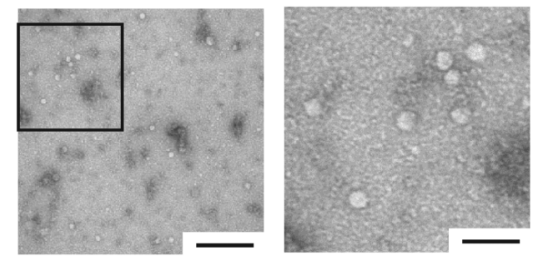

$\mathrm{C}$

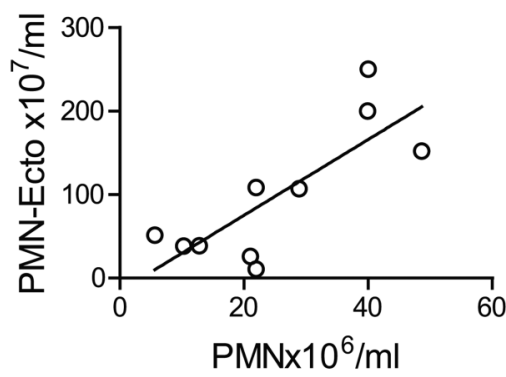

$p=0.006$

$r^{2}=0.628$

$\rightarrow$ linear regression

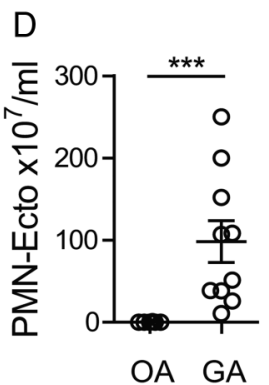

E

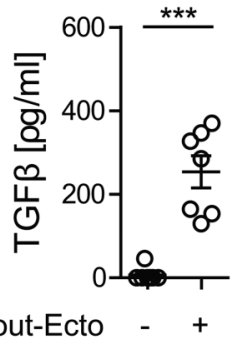

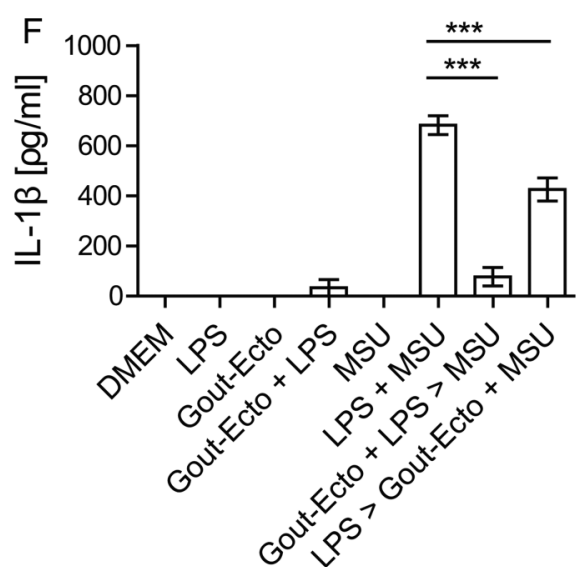
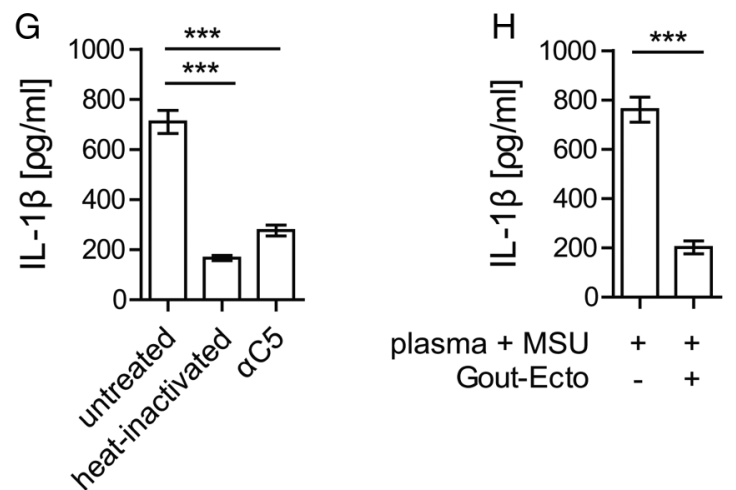

plasma + MSU + +

Gout-Ecto - +

Figure 6 PMN-Ecto present in gout exudates in humans have immunosuppressive properties. PMN-Ecto were isolated from joint aspirates of patients undergoing a gout attack. (A) Ecto were isolated from joint aspirates as indicated in methods and characterised and counted by flow cytometry. Annexin V, anti-CD66b and anti-myeloperoxidase (MPO) antibodies identified them to be of neutrophil origin. Counting was performed using microbeads. (B) Transmission electron microscopy of PMN-Ecto. Size bar $1 \mu \mathrm{m}$ (left) and $100 \mathrm{~nm}$ (right). (C) Correlation between the number of infiltrating PMN and PMN-Ecto found in gout exudates. Each dot represents a single patient. (D) Concentration of PMN-Ecto in gout (gouty arthritis, GA) and osteoarthritis (OA) exudates. (E-H) PMN-Ecto isolated from joint aspirates are functional in vitro. (E) Release of TGF $\beta$ by human

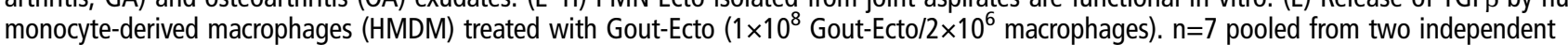
experiments. (F) Suppression of IL-1 $\beta$ release by HMDM treated with Gout-Ecto following the in vitro protocol outlined in figure $1 D$. (G) IL-1 $\beta$ release upon $14 \mathrm{~h}$ stimulation of HMDM with $100 \mu \mathrm{g} / \mathrm{mL}$ monosodium urate (MSU) in $25 \%$ human plasma (untreated), heat inactivated plasma (heat inactivated) or $\mathrm{C} 5$-blocked plasma $(\alpha \mathrm{C} 5)$. ( $\mathrm{F}$ and $\mathrm{G}) \mathrm{n}=6$ pooled from three independent experiments. (H) Suppression of IL-1 $\beta$ release by HMDM treated with Gout-Ecto and stimulated as outlined in $(G) .{ }^{* * *} p<0.001$. Mean $\pm S E M$ is shown. 
physiological and the amount was set at the maximum number of PMN-Ecto recovered from the peritoneum after MSU stimulation. Since ectosomes bind back to cells ${ }^{9}$ and are continuously cleared, ${ }^{25}$ the recovery of $1.5 \times 10^{7}$ PMN-Ecto is likely an underestimation of the total amount of ectosomes shed during the course of MSU peritonitis.

Previous studies have already suggested that PS expressed on apoptotic cells ${ }^{3-5} 7$ and ectosomes ${ }^{26}$ contribute to the resolution of inflammation. PS has been shown to mediate antiinflammatory signals via the MerTK receptor. ${ }^{5}$ MerTK activation leads to induction of SOCS $3^{6}$ and in turn to suppression of TLR-induced cytokine release. ${ }^{5} 7{ }^{15}$ In accordance with these studies, we could confirm that PS-expressing ectosomes suppress LPS priming of the NLRP3 inflammasome in vitro. LPS priming, however, is not required for the inflammatory response to MSU in vivo and is therefore merely used to model inflammasome activation in vitro. ${ }^{16}$ In addition to activating the inflammasome, MSU crystals activate the complement cascade by assembling a C5 convertase on their surface. ${ }^{17} 27$ We could confirm that C5a generated by MSU is the main inflammasome priming agent in gout in vivo. ${ }^{28}$ The anti-inflammatory effect of PMN-Ecto was therefore not limited to the inhibition of TLR stimuli, but extended to C5a and C5aR signalling as well. Furthermore, C5a was able to prime the inflammasome and limit its activation by inducing the release of PMN-Ecto, thus forming the basis of an autoregulatory negative feedback loop in gout. ${ }^{9}$ This mechanism may apply to other crystal arthropathies such as those caused by calcium pyrophosphate dihydrate and hydroxyapatite crystals, since Ecto suppress calcium pyrophosphate dihydrate and hydroxyapatite-induced IL- $1 \beta$ release as well (see online supplementary figure S1C).

Whereas studies using liposomes suggested that PS-positive vesicles could downregulate inflammation, involvement of PS was confirmed in mice deficient for the PS receptor MerTK. Furthermore, the higher degree of inflammation in $\mathrm{MerTK}^{-/-}$compared with WT mice suggested that PS expressed on Ecto provides baseline suppression in gouty inflammation. Of note, high expression of SOCS3 in synovial tissue has been found in patients during the acute phase of gout, ${ }^{8}$ further supporting the notion that the PS-MerTK axis plays a role in limiting gouty inflammation.

TGF $\beta$ is considered to play an active role in the resolution of gout. ${ }^{8} 29{ }^{30}$ Levels of TGF $\beta$ have been found to progressively increase in synovial fluids of patients with gout after an attack, suggesting that TGF $\beta$ may be involved in late phases of resolution. ${ }^{82}$ We demonstrated that ectosomes trigger the release of TGF $\beta$ in vitro and in vivo. These findings are consistent with other studies that have shown release of TGF $\beta$ by macrophages ${ }^{4}$ or neutrophils ${ }^{3}$ in response to apoptotic cells and ectosomes. ${ }^{26}$ In vivo, however, neither treatment with recombinant mouse TGF $\beta 1$ (figure 5J), nor the release of TGF $\beta$ by ectosomes in the absence of PS-MerTK activation (figure 5G) was sufficient to control the acute inflammatory response to MSU. Furthermore, PS-liposomes alone could reproduce the ectosome effect without inducing the release of TGF $\beta$ (figure 3C-D, figure $5 \mathrm{H}$ ) and TGF $\beta$ blocking did not compromise the ability of BM-Ecto to suppress inflammation (figure 5I). These findings argue against a role for TGF $\beta$ in the early phase of gout. Given that patients with gout with TGF $\beta$ polymorphisms frequently progress to more advanced disease states, TGF $\beta$ may play a role in chronic gout and affect the rate of progression rather than resolution of acute attacks. ${ }^{31}$ Aside from TGF, IL-10 and IL-1Ra have also been associated with anti-inflammatory effects, but they were not induced by ectosomes in the MSU peritonitis model (see online supplementary figure S1D-F). ${ }^{32-34}$
Recently, the resolution of gout has been associated with another phenomenon of neutrophil activation, the release of neutrophil extracellular traps (NETs). ${ }^{35}$ NETs were shown to degrade proinflammatory chemokines by clustering serine proteases such as proteinase 3 and neutrophil elastase. In our model of acute gout we could not measure significant increase of cell-free DNA or NETs (see online supplementary figure S1G) and the treatment with DNAse I did not significantly alter the outcome of MSU-induced inflammation (see online supplementary figure S1H). Although NETs did not play a role in this model, the degradation of chemokines by proteases may still be relevant, since PMN-Ecto actively recruit proteinase 3 and neutrophil elastase from fluid phase to their surface. $^{9}{ }^{36}$ PMN-Ecto may not only suppress IL- $1 \beta$ release upon MSU stimulation, but potentially also degrade IL-1 $\beta$ once it has been released. This could possibly explain the higher efficacy of PMN-Ecto compared with liposomes, which carry no enzymes (figure $3 \mathrm{~B}, \mathrm{D}$ ).

A limitation of our study was the exclusive use of the MSU peritonitis model. It would be of interest to assess whether ectosomes elicit similar responses in alternate models of gout, such as the intra-articular or air-pouch model, which are known to have various degrees of NLRP3, ASC and caspase 1 involvement in IL-1 $\beta$ release. ${ }^{37}$

The motivation behind this work was to better understand the self-limiting nature of gout. This study supports the notion that resolution of gout is initiated with the release of PMN-Ecto early on during neutrophil activation. In a broader context, PMN-Ecto release may limit excessive inflammation in response to exogenous and endogenous danger signals and their pathophysiological relevance likely extends to conditions other than gout. $^{38}$

Acknowledgements The authors thank Prof Petr Broz and Dr Etienne Meunier (Biozentrum, University of Basel) for technical assistance, Prof van den Broek and Dr Laura Surace (Institute of Experimental Immunology, University of Zürich) for providing the $\mathrm{C} \mathrm{aR}^{-/-}$mice, Prof Ed Palmer (Department of Biomedicine, University Hospital Basel) for helpful discussions and Vesna Olivieri (Microscopy Core Facility, Biozentrum, University of Basel) for performing electron microscopy.

Contributors $A C$ designed, performed and analysed all experiments, wrote the manuscript. BA provided clinical samples from the COUGAR gout cohort. DZ and JAS supervised the project and wrote the manuscript.

Funding JAS was supported by the Swiss National Science Foundation (320030_146255/1); DZ was supported by Fondation Machaon, Geneva, Switzerland; BA was supported by Hedwig Widmer Stiftung, Zurich and Freie Akademische Geselschaft, Basel, Switzerland.

Competing interests None declared.

Ethics approval Ethikkommission Beider Basel, EKBB no. 75/12.

Provenance and peer review Not commissioned; externally peer reviewed.

Data sharing statement AC, DZ and JAS had full access to all data in the study and take responsibility for the integrity of the data and the accuracy of the data analysis. No additional data are available.

Open Access This is an Open Access article distributed in accordance with the terms of the Creative Commons Attribution (CC BY 4.0) license, which permits others to distribute, remix, adapt and build upon this work, for commercial use, provided the original work is properly cited. See: http://creativecommons.org/ licenses/by/4.0/

\section{REFERENCES}

1 Martinon F, Pétrilli V, Mayor A, et al. Gout-associated uric acid crystals activate the NALP3 inflammasome. Nature 2006;440:237-41.

2 Rose DM, Sydlaske AD, Agha-Babakhani A, et al. Transglutaminase 2 limits murine peritoneal acute gout-like inflammation by regulating macrophage clearance of apoptotic neutrophils. Arthritis Rheum 2006;54:3363-71.

3 Steiger $S$, Harper JL. Neutrophil cannibalism triggers transforming growth factor $\beta 1$ production and self regulation of neutrophil inflammatory function in monosodium 
urate monohydrate crystal-induced inflammation in mice. Arthritis Rheum 2013;65:815-23.

4 Huynh ML, Fadok VA, Henson PM. Phosphatidylserine-dependent ingestion of apoptotic cells promotes TGF-beta1 secretion and the resolution of inflammation. J Clin Invest 2002;109:41-50.

5 Yi Z, Li L, Matsushima GK, et al. A novel role for c-Src and STAT3 in apoptotic cell-mediated MerTK-dependent immunoregulation of dendritic cells. Blood 2009:114:3191-8

6 Rothlin CV, Ghosh S, Zuniga El, et al. TAM receptors are pleiotropic inhibitors of the innate immune response. Cell 2007;131:1124-36.

7 Sen P, Wallet MA, Yi Z, et al. Apoptotic cells induce Mer tyrosine kinase-dependent blockade of NF-kappaB activation in dendritic cells. Blood 2007;109:653-60.

8 Chen YH, Hsieh SC, Chen WY, et al. Spontaneous resolution of acute gouty arthritis is associated with rapid induction of the anti-inflammatory factors TGF $\beta 1, \mathrm{IL}-10$ and soluble TNF receptors and the intracellular cytokine negative regulators CIS and SOCS3. Ann Rheum Dis 2011;70:1655-63.

9 Gasser 0 , Hess C, Miot $S$, et al. Characterisation and properties of ectosomes released by human polymorphonuclear neutrophils. Exp Cell Res 2003;285:243-57.

10 Eken C, Gasser O, Zenhaeusern G, et al. Polymorphonuclear neutrophil-derived ectosomes interfere with the maturation of monocyte-derived dendritic cells. $J$ Immunol 2008:180:817-24.

11 Broz P, Monack DM. Measuring inflammasome activation in response to bacterial infection. Methods Mol Biol 2013;1040:65-84.

12 Bauernfeind FG, Horvath G, Stutz A, et al. Cutting edge: NF-kappaB activating pattern recognition and cytokine receptors license NLRP3 inflammasome activation by regulating NLRP3 expression. J Immunol 2009;183:787-91.

13 Guarda G, Dostert C, Staehli F, et al. T cells dampen innate immune responses through inhibition of NLRP1 and NLRP3 inflammasomes. Nature 2009;460:269-73.

14 Gross 0, Thomas CJ, Guarda G, et al. The inflammasome: an integrated view. Immunol Rev 2011;243:136-51.

15 Eken C, Martin PJ, Sadallah S, et al. Ectosomes released by polymorphonuclear neutrophils induce a MerTK-dependent anti-inflammatory pathway in macrophages. J Biol Chem 2010;285:39914-21.

16 Chen CJ, Shi Y, Hearn A, et al. MyD88-dependent IL-1 receptor signaling is essential for gouty inflammation stimulated by monosodium urate crystals. J Clin Invest 2006;116:2262-71.

17 Russell IJ, Mansen C, Kolb LM, et al. Activation of the fifth component of human complement (C5) induced by monosodium urate crystals: C5 convertase assembly on the crystal surface. Clin Immunol Immunopathol 1982;24:239-50.

18 Kastl SP, Speidl WS, Kaun C, et al. The complement component C5a induces the expression of plasminogen activator inhibitor-1 in human macrophages via NF-kappaB activation. J Thromb Haemost 2006:4:1790-7

19 Pan ZK. Anaphylatoxins C5a and C3a induce nuclear factor kappaB activation in human peripheral blood monocytes. Biochim Biophys Acta 1998;1443:90-8

20 Laudisi F, Spreafico R, Evrard M, et al. Cutting edge: the NLRP3 inflammasome links complement-mediated inflammation and IL-1 $\beta$ release. J Immunol 2013;191:1006-10.
21 Martin WJ, Walton M, Harper J. Resident macrophages initiating and driving inflammation in a monosodium urate monohydrate crystal-induced murine peritoneal model of acute gout. Arthritis Rheum 2009;60:281-9.

22 Scanu A, Oliviero F, Ramonda $R$, et al. Cytokine levels in human synovial fluid during the different stages of acute gout: role of transforming growth factor $\beta 1$ in the resolution phase. Ann Rheum Dis 2012;71:621-4.

23 Doherty TA, Soroosh $\mathrm{P}$, Khorram N, et al. The tumor necrosis factor family member LIGHT is a target for asthmatic airway remodeling. Nat Med 2011;17:596-603.

24 Slobodin G, Kaly L, Peri R, et al. Higher expression of latency-associated peptide on the surface of peripheral blood monocytes in patients with rheumatoid arthritis may be protective against articular erosions. Inflammation 2013;36:1075-8.

25 Willekens FL, Werre JM, Kruijt JK, et al. Liver Kupffer cells rapidly remove red blood cell-derived vesicles from the circulation by scavenger receptors. Blood 2005; 105:2141-5.

26 Gasser 0, Schifferli JA. Activated polymorphonuclear neutrophils disseminate anti-inflammatory microparticles by ectocytosis. Blood 2004;104:2543-8.

27 Tramontini N, Huber C, Liu-Bryan R, et al. Central role of complement membrane attack complex in monosodium urate crystal-induced neutrophilic rabbit knee synovitis. Arthritis Rheum 2004;50:2633-9.

28 An LL, Mehta P, Xu L, et al. Complement C5a potentiates uric acid crystal-induced IL-1 $\beta$ production. Eur J Immunol 2014;44:3669-79.

29 Yagnik DR, Evans BJ, Florey 0, et al. Macrophage release of transforming growth factor beta 1 during resolution of monosodium urate monohydrate crystal-induced inflammation. Arthritis Rheum 2004;50:2273-80.

30 Lioté $F$, Prudhommeaux F, Schiltz C, et al. Inhibition and prevention of monosodium urate monohydrate crystal-induced acute inflammation in vivo by transforming growth factor beta1. Arthritis Rheum 1996;39:1192-8.

31 Chang SJ, Chen CJ, Tsai FC, et al. Associations between gout tophus and polymorphisms $869 \mathrm{~T} / \mathrm{C}$ and $-509 \mathrm{C} / \mathrm{T}$ in transforming growth factor beta1 gene. Rheumatology (Oxford) 2008;47:617-21.

32 Greenhill CJ, Jones GW, Nowell MA, et al. Interleukin-10 regulates the inflammasome-driven augmentation of inflammatory arthritis and joint destruction. Arthritis Res Ther 2014;16:419.

33 Aouba A, Deshayes S, Frenzel $L$, et al. Efficacy of anakinra for various types of crystal-induced arthritis in complex hospitalized patients: a case series and review of the literature. Mediators Inflamm 2015;2015:792173.

34 So A, De Smedt T, Revaz S, et al. A pilot study of IL-1 inhibition by anakinra in acute gout. Arthritis Res Ther 2007;9:R28.

35 Schauer C, Janko C, Munoz LE, et al. Aggregated neutrophil extracellular traps limit inflammation by degrading cytokines and chemokines. Nat Med 2014;20:511-17.

36 Hess C, Sadallah S, Schifferli JA. Induction of neutrophil responsiveness to myeloperoxidase antibodies by their exposure to supernatant of degranulated autologous neutrophils. Blood 2000;96:2822-7.

37 Joosten LA, Ea HK, Netea MG, et al. Interleukin-1beta activation during acute joint inflammation: a limited role for the NLRP3 inflammasome in vivo. Joint Bone Spine 2011;78:107-10

38 Nauseef WM, Borregaard N. Neutrophils at work. Nat Immunol 2014;15:602-11. 